\title{
Review Article \\ Recent Progress in Deciphering the Etiopathogenesis of Primary Membranous Nephropathy
}

\author{
Andreas Kronbichler, ${ }^{1}$ Jun Oh, ${ }^{2}$ Björn Meijers, ${ }^{3,4}$ Gert Mayer, ${ }^{1}$ and Jae Il Shin ${ }^{5}$ \\ ${ }^{1}$ Department of Internal Medicine IV (Nephrology and Hypertension), Medical University Innsbruck, Anichstraße 35, \\ 6020 Innsbruck, Austria \\ ${ }^{2}$ Pediatric Nephrology, University Medical Center Hamburg-Eppendorf, Martinistraße 52, 20246 Hamburg, Germany \\ ${ }^{3}$ Department of Nephrology, UZ Leuven, Leuven, Belgium \\ ${ }^{4}$ Department of Immunology and Microbiology, KU Leuven, Leuven, Belgium \\ ${ }^{5}$ Department of Pediatrics, Yonsei University College of Medicine, Severance Children's Hospital, Seoul, Republic of Korea
}

Correspondence should be addressed to Andreas Kronbichler; andreas.kronbichler@i-med.ac.at

Received 27 February 2017; Accepted 18 July 2017; Published 17 August 2017

Academic Editor: Christophe Duranton

Copyright ( 2017 Andreas Kronbichler et al. This is an open access article distributed under the Creative Commons Attribution License, which permits unrestricted use, distribution, and reproduction in any medium, provided the original work is properly cited.

\begin{abstract}
Primary membranous nephropathy $(\mathrm{MN})$ is the leading cause of nephrotic syndrome in adults. Discovery of several antibodies has contributed to an increased understanding of MN. Antibodies against the M-type phospholipase A2 receptor (PLA2R) are present in $50-100 \%$ with primary $\mathrm{MN}$ and are associated with a lower frequency of spontaneous remission. High levels are linked with a higher probability of treatment resistance, higher proteinuria, and impaired renal function, as well as a more rapid decline of kidney function during follow-up. Immunologic remission precedes reduction of proteinuria by months. Pretransplant evaluation of PLA2R antibodies is warranted to predict recurrence of disease following renal transplantation. Several risk alleles related to the PLA2R1 gene and within the HLA loci have been identified, whereas epitope spreading of PLA2R may predict treatment response. More recently, thrombospondin type 1 domain-containing 7A (THSD7A) antibodies have been discovered in primary MN. Several other rare antigens have been described, including antibodies against neutral endopeptidase as a cause of antenatal MN and circulating cationic bovine serum albumin as an antigen with implications in childhood MN. This review focuses on the progress with a special focus on diagnostic accuracy, predictive value, and treatment implications of the established and proposed antigens.
\end{abstract}

\section{Introduction}

Recent research in the field has unraveled important pathogenetic insights in the onset of primary membranous nephropathy $(\mathrm{MN})$. Distinction between primary and secondary cases (mainly autoimmune disorders, infections, medications, and malignancy), characterized by a recognizable etiology, is pivotal. A clear difference from a histopathologic perspective is difficult, but absence of phospholipase A2 receptor (PLA2R) glomerular staining, preponderance of IgG1-3, and presence of extensive mesangial or subendothelial electron dense deposits may have a value in the diagnosis of secondary cases $[1,2]$. While IgG1 is the predominant IgG subclass observed in secondary cases (60\% of biopsies), IgG4 is predominantly found in subjects with primary $\mathrm{MN}$ ( $76 \%$ of biopsies) [3]. The exact role of the least represented IgG subclass is not completely understood, but it may exhibit distinct roles compared to IgG1 in allergy and tumor growth [4], and has attracted considerable interest due to increased incidence and prevalence of IgG4-related disease $[5,6]$.

Globally, MN represents the leading cause of nephrotic syndrome, with a reported annual incidence ranging from $0.2 / 100.000$ /year to $1.4 / 100.000$ /year and an overall estimate of 1.2/100.000/year [7]. A male predominance has been reported in most studies $[7,8]$, prognosis is related to renal function decline, and outcome tends to be better in females [8]. In general, patients present with a greater degree of proteinuria compared to those with focal segmental 
glomerulosclerosis (FSGS) and minimal change disease (MCD), but renal survival is assumed to be better compared to those having FSGS [9-11]. In line with FSGS [12], recurrence of disease after kidney transplantation takes place in approximately $35 \%$ of patients [13]. Recurrence is supposed to occur due to a yet-to-be-defined circulatory factor in FSGS, while progress in the understanding of MN has led to identification of circulatory factors leading to disease onset. While neonatal disease is associated with antibodies against neutral endopeptidase [14], M-type phospholipase A2 receptor antibody (PLA2R-Ab) [15], and thrombospondin type 1 domain-containing 7A (THSD7A) [16] autoantibodies are responsible for approximately $80 \%$ of primary $\mathrm{MN}$ cases. The aim of this review is to summarize recent findings in primary $\mathrm{MN}$, focusing on implications of identified antibodies as well as their diagnostic accuracy and therapeutic impact.

\section{M-Type Phospholipase A2 Receptor Autoantibodies and Glomerular Phospholipase A2 Receptor in Primary Membranous Nephropathy}

PLA2R-Abs were first identified by Western-blotting and subsequent mass spectrometry in 26 out of 37 (70\%) patients with primary MN. The $185-\mathrm{kD}$ protein was absent in secondary $\mathrm{MN}$ as well as other proteinuric diseases (diabetic kidney disease, FSGS, or other autoimmune disorders). Moreover, samples reacting with the glycoprotein recognized recombinant PLA2R in vitro and PLA2R could be detected in human glomeruli. Glomerular deposits of patients with primary $\mathrm{MN}$ had a predominance of IgG4-subclass antibodies, and PLA2R colocalized with IgG4 in glomeruli [15]. A subsequent large multicenter study from Europe including 117 patients revealed presence of PLA2R-Ab in 74\% and 72\% using an immunofluorescence test (IFT) or ELISA, which increased to $76 \%$ when positivity in either test was considered as positive finding. The IgG4 subclass was present in $69 \%$ of all patients and correlated best with antibody positivity measured by IFT or ELISA [17]. Analysis of PLA2R staining in MN revealed positivity in $69 \%$ of 88 patients and was accompanied by PLA2R-Ab positivity in 60/61 patients (different time points of blood sampling). Enhanced PLA2R staining was exclusively found in primary MN and, of note, IgG4 staining was present in $95 \%$ of primary $\mathrm{MN}$ and in only $20 \%$ with secondary causes [18]. A study from the Netherlands investigated 18 samples during active disease and found a frequency of $77.8 \%$ PLA2R-related MN in their cohort [19]. In a prospective study by Hoxha and colleagues, PLA2R-Ab were measured in treatment-naïve patients. PLA2R-Ab were detected in $133 / 163(81.6 \%)$ patients [20].

Several studies from Asia investigated presence of PLA2R-Ab and glomerular PLA2R staining. In a Korean study, 69/100 patients (69\%) had detectable PLA2R-Ab as assessed by Western blot [21]. PLA2-related MN as detected either by IFT, by ELISA, or by glomerular PLA2R staining was reported in 94/114 (82.5\%) in a study from India [22]. A study from China including consecutive patients with primary $\mathrm{MN}$ reported a frequency of $64.6 \%(53 / 82)$ [23]. In contrast to this low frequency of antibody positivity, another study from China found PLA2R-Ab in $49 / 60$ patients (81.7\%). When decreasing the dilution of samples for Western blot analysis, 10 of the remaining samples had low titers, albeit with a weaker strength compared to the other positive samples [24]. A larger study including 572 patients found detectable PLA2R-Ab in $68.5 \%(n=392)$ as assessed by ELISA. Among the antibody negative cases $(n=180)$, glomerular staining was present in $70.6 \%(n=127)$ [25]. Similar high frequency of PLA2R-MN (92.2\%) was reported from another Chinese study analyzing 179 primary MN patients [26]. In Japanese patients, the prevalence of PLA2R-Ab may be lower compared to the rest of the world. A retrospective analysis of patients with 100 primary MN cases (after screening for clinical or laboratory signs of a secondary cause) revealed positivity in $53 \%$ of patients, with a higher prevalence observed in these subjects with nephrotic range proteinuria (61\% versus $43 \%$ ) [27]. Another study found PLA2R-Ab in 50\% of their patients, whereas similar frequency of positive glomerular PLA2R staining could be detected in two cohorts (52.6 and $52.7 \%$, resp.) $[28,29]$. The respective studies reporting adultonset primary MN are summarized in Table 1.

Analysis of PLA2R-Ab screening during remission showed a limited predictive value to identify PLA2R-related MN, with 8 out of 37 (22\%) samples testing positive, whereas PLA2R was found in 22 of 37 (59\%) corresponding biopsies [43]. PLA2R-Ab levels have been examined in childhood MN and were present to a lesser extent in a pediatric population, ranging from $2 / 34(5.9 \%)$ to $10 / 22(45.5 \%)$ patients reported from Japan and Northern America [44, 45]. In contrast, Kumar and coworkers reported PLA2-related MN in 15/18 $(83.3 \%)$ of adolescents with primary MN [46].

In general, discrimination between primary and secondary cases is pivotal. In individual studies, a substantial heterogeneity has been reported. To better characterize PLA2R-Ab as a marker for primary MN, two meta-analyses were conducted. The more recent study by Dai and colleagues included 19 studies with 1160 patients. PLA2R-Ab positivity yielded a sensitivity of 0.68 , a specificity of 0.97 , a diagnostic odds ratio of 73.75, and an area under the curve of 0.82 when comparing primary and secondary $\mathrm{MN}$. The respective values for glomerular PLA2R staining were 0.78, 0.91, 34.70, and 0.84 for the same parameters without heterogeneity [47].

The GEMRITUX trial comparing nonimmunosuppressive antiproteinuric treatment (NIAT) with rituximab against NIAT alone found PLA2R-Ab in three subjects who were initially found to be negative [38]. In line, "seroconversion" has been reported in a recent case series and a "kidney in the sink"-hypothesis has been generated, discussing the role of a PLA2R-Ab buffer capacity exhaustion of the kidney leading to antibody detection at a later stage [48]. In contrast to this assumption, a case report reported presence of PLA2R-Ab in a patient eight months ahead of MN presentation. PLA2R-Ab was measured during a thrombophilia work-up [49].

Taken together, the ideal time point of sample collection is at the time of kidney biopsy and ahead of treatment initiation, which may account for misclassification as PLA2R$\mathrm{Ab}$ negative $\mathrm{MN}$ in some cases. Detection of serum PLA2R$\mathrm{Ab}$ by diverse commercial measures is reliable, but cases of 
TABLE 1: A summary of studies investigating the prevalence of either phospholipase A2 receptor antibodies, glomerular phospholipase A2 receptor staining, or both in primary membranous nephropathy. ELISA (Enzyme-Linked Immunosorbent Assay), IFT (Indirect Immunofluorescence Test), PLA2R (phospholipase a2 receptor), PLA2R-Ab (phospholipase a2 receptor antibody), WB (Western blot). ${ }^{*}$ Dilution of serum samples was decreased to $1: 10$, and exposure time was increased to 10 minutes.

\begin{tabular}{|c|c|c|c|c|c|}
\hline Reference & Country & $\begin{array}{l}\text { Prevalence } \\
\text { (PLA2R-Ab) }\end{array}$ & Detection method & $\begin{array}{c}\text { Prevalence (PLA2R } \\
\text { staining) }\end{array}$ & Predominant IgG subclass \\
\hline [15] & USA & $26 / 37(70 \%)$ & WB & & \\
\hline [17] & Netherlands, France, UK & $\begin{array}{l}87 / 117(74 \%) \\
84 / 117(72 \%) \\
\end{array}$ & $\begin{array}{c}\text { IFT, } \\
\text { ELISA }\end{array}$ & & IgG4 (81/87, serum) \\
\hline$[18]$ & Germany & $60 / 88(68 \%)$ & IFT & $61 / 88(69 \%)$ & IgG4 (58/61, biopsy) \\
\hline [19] & Netherlands & $14 / 18(78 \%)$ & WB & & IgG4 (14/14, serum) \\
\hline$[20]$ & Germany & $133 / 163(82 \%)$ & IFT, ELISA & & \\
\hline [21] & Korea & $69 / 100(69 \%)$ & WB & & \\
\hline$[22]$ & India & $\begin{array}{l}75 / 114(65 \%) \\
76 / 114(67 \%)\end{array}$ & $\begin{array}{c}\text { IFT, } \\
\text { ELISA }\end{array}$ & $86 / 114(75 \%)$ & \\
\hline$[23]$ & China & $\begin{array}{l}53 / 82(65 \%) \\
51 / 82(62 \%)\end{array}$ & $\begin{array}{c}\text { IFT, } \\
\text { ELISA }\end{array}$ & & \\
\hline$[24]$ & China & $\begin{array}{c}49 / 60(82 \%) \\
59 / 60(98 \%)^{*}\end{array}$ & WB & & \\
\hline$[25]$ & China & $392 / 572(69 \%)$ & ELISA & $514 / 572(90 \%)$ & $\begin{array}{c}\text { IgG4 (97.7\% (PLA2R-Ab/R } \\
+/+), 98.4 \%(\text { PLA2R-Ab/R } \\
-/+)) \\
\end{array}$ \\
\hline [26] & China & & & $165 / 179(92 \%)$ & $\operatorname{IgG} 4(167 / 179,93 \%)$ \\
\hline [27] & Japan & $53 / 100(53 \%)$ & WB & & \\
\hline [28] & Japan & $\begin{array}{l}19 / 38(50 \%) \\
19 / 38(50 \%) \\
\end{array}$ & $\begin{array}{c}\text { IFT, } \\
\text { ELISA }\end{array}$ & $20 / 38(53 \%)$ & IgG4 (20/20, biopsy) \\
\hline [29] & Japan & & & $29 / 55(53 \%)$ & $\operatorname{IgG4}(28 / 29$, biopsy) \\
\hline
\end{tabular}

PLA2R-related MN may be missed by antibody detection only. Thus, staining for PLA2R antigen in the kidney should be considered to overcome this limitation. A preponderance of IgG4 is found in cases with PLA2R-related MN in serum and kidney specimens. Presence of PLA2R-Ab show a lower prevalence in Japan (around 50\%) and in early-onset $\mathrm{MN}$ compared to other investigated cohorts (PLA2R-Ab positivity ranging from 65 to almost 100\%). While PLA2R-Ab detection and predominant IgG4 presence may be considered a good measure to discriminate primary from secondary causes of $\mathrm{MN}$ in Caucasians, this is of lower validity in Chinese patients as shown by the recent meta-analyses.

2.1. Secondary Membranous Nephropathy and PLA2R Ab or PLA2R Staining Positivity. Initial studies have reported PLA2R-Ab positivity occurring in primary $\mathrm{MN}$ only. With the increasing body of evidence, secondary $\mathrm{MN}$ forms positive for either PLA2R-Ab or glomerular PLA2R staining have been identified.

In a cohort of patients with membranous lupus nephritis (LN), PLA2R-Abs were undetectable [30]. This is in line with Japanese studies reporting PLA2R-Ab positivity exclusively in patients with primary MN [27] or absence of either PLA2R$\mathrm{Ab}$ or glomerular staining for PLA2R [28], whereas a low frequency (5.4\%) could be observed in another analysis (one case HBV-associated and one malignancy-related) [29]. Positive PLA2R staining had a sensitivity of $75 \%$ and a specificity of $83 \%$ for primary $\mathrm{MN}$ in a large US study ( 85 primary and 80 secondary cases). Predominant secondary causes were hepatitis C (7/11, 64\%), sarcoidosis $(3 / 4,75 \%)$, and malignancy $(3 / 12,25 \%)$, whereas positive staining was found to a lesser extent in patients with autoimmune diseases $(1 / 46,2 \%)$. Interestingly, all PLA2R positive secondary $\mathrm{MN}$ cases showed IgG4-predominant staining [31]. A French nationwide survey retrospectively analyzed kidney biopsies from patients with sarcoidosis and found PLA2R antigen in immune deposits in all patients. Serial serum samples were obtained in two patients and circulating PLA2R-Ab levels were in accordance with sarcoidosis activity and both, PLA2R-Ab and PLA2R, were predominantly related to the IgG4 subclass [50].

In a Chinese study, $84 \%$ of 102 tissue samples of patients with primary MN were PLA2R positive. Among secondary causes, one out of 38 patients with class V LN and 64\% with hepatitis B virus-associated MN (HBV-MN) showed positive PLA2R staining [32]. Of 22 cases with secondary MN (HBV-MN, LN or both), $36.4 \%$ (8/22) had detectable PLA2R-Ab [23]. Analysis of 24 patients with psoriasis and MN revealed PLA2R-Ab and PLA2R positivity in 7 (29.2\%) subjects. Among the patients with PLA2R-positivity, a majority showed IgG4 predominance on immunofluorescence (IF) [33]. Secondary causes of MN were accompanied by detectable PLA2R-Ab in a minority of patients in a Chinese study, including $1 / 20$ positive cases with $\mathrm{LN}, 1 / 16$ with HBV-MN, and 3/10 with malignancy-associated MN. In line with other observations, IgG4 was the predominant PLA2Rreactive subclass [24]. A Korean study found PLA2R-Ab in 
TABLE 2: Secondary causes of membranous nephropathy and the prevalence of either PLA2R-Ab or positive glomerular PLA2R staining are summarized. AID (autoimmune diseases), ELISA (Enzyme-Linked Immunosorbent Assay), IgG4-RD (IgG4-related disease), IFT (Indirect Immunofluorescence Test), LN (lupus nephritis), PLA2R (Phospholipase A2 Receptor), PLA2R-Ab (phospholipase A2 receptor antibody), and WB (Western blot). ${ }^{*}$ Notably, 25/39 (64\%) of patients with hepatitis B-associated MN had detectable PLA2R staining, whereas PLA2R positivity was found in 1/38 (2.6\%) of lupus nephritis cases. ${ }^{* 1}$ By decreasing the dilution, 2 additional cases of hepatitis B-associated MN were considered positive.

\begin{tabular}{|c|c|c|c|c|c|c|}
\hline Reference & Country & $\begin{array}{c}\text { Prevalence } \\
\text { (PLA2R-Ab) }\end{array}$ & $\begin{array}{l}\text { Detection } \\
\text { method }\end{array}$ & $\begin{array}{c}\text { Prevalence (PLA2R } \\
\text { staining) }\end{array}$ & $\begin{array}{c}\text { Predominant IgG } \\
\text { subclass }\end{array}$ & Entities \\
\hline$[15]$ & USA & $0 / 8(0 \%)$ & WB & & & LN, hepatitis B \\
\hline$[30]$ & Sweden & $0 / 25(0 \%)$ & IFT & & & $\mathrm{LN}$ \\
\hline [27] & Japan & $0 / 31(0 \%)$ & WB & & & $\begin{array}{c}\text { AID, cancer, drugs, hepatitis B, } \\
\text { others }\end{array}$ \\
\hline [28] & Japan & $0 / 21(0 \%)$ & IFT, ELISA & & & LN, cancer, hepatitis B \\
\hline [29] & Japan & & & $2 / 37(5 \%)$ & $\operatorname{IgG} 4$ (2/2, biopsy) & $\begin{array}{l}\text { AID, cancer, drugs, hepatitis B, } \\
\text { hepatitis C, thyroiditis }\end{array}$ \\
\hline$[31]$ & USA & & & $14 / 80(18 \%)$ & IgG4 (6/6, biopsy) & $\begin{array}{c}\text { AID, cancer, hepatitis B, hepatitis C, } \\
\text { HIV, syphilis }\end{array}$ \\
\hline [32] & China & & & $26 / 77(34 \%)^{*}$ & & LN, hepatitis B \\
\hline [23] & China & $8 / 22(36 \%)$ & IFT, ELISA & & & LN, hepatitis B \\
\hline [33] & China & $7 / 24(29 \%)$ & IFT & $7 / 24(29 \%)$ & IgG4 (5/6, biopsy) & psoriasis \\
\hline$[24]$ & China & $\begin{array}{c}5 / 46(11 \%) \\
7 / 46(15 \%)^{* 1}\end{array}$ & WB & & IgG4 & LN, cancer, hepatitis B \\
\hline [21] & Korea & $2 / 9(22 \%)$ & WB & & & LN, cancer, hepatitis B, hepatitis C \\
\hline [26] & China & & & $2 / 69(3 \%)$ & $\operatorname{IgG} 4$ (2/2, biopsy) & LN, cancer, hepatitis B, IgG4-RD \\
\hline
\end{tabular}

two out of nine patients with secondary MN (1 HBV and 1 malignancy-associated) and both showed predominant IgG4 immunostaining [21]. Positive PLA2R-staining was found in $2 / 26(7.7 \%)$ of patients with HBV-MN and 2/2 (100\%) presenting with malignancy. All patients with secondary causes and detectable glomerular PLA2R staining had predominant IgG4 deposition [26]. The respective findings are summarized in Table 2.

A preponderance of IgG4 staining on IF is assumed to be present in primary MN. As discussed by De Vriese and colleagues, the question arises whether some of these cases represent true secondary $\mathrm{MN}$ or rather PLA2R-related $\mathrm{MN}$ with coincident secondary diseases [51]. A higher frequency of secondary causes associated with PLA2R-Ab or PLA2R staining has been reported in Chinese studies, especially cases with hepatitis B-associated $\mathrm{MN}$ known to be endemic in China. Among the diseases with antibody positivity or positive staining, hepatitis B- or C-associated MN, malignancyassociated $\mathrm{MN}$, and sarcoidosis emerged to be predominantly found.

\subsection{Correlation of PLA2R-Ab Titers with Clinical Characteris-} tics. In a study including 101 primary MN patients, baseline creatinine levels appeared to be higher in PLA2R-Ab positive patients, whereas baseline rate of proteinuria correlated with PLA2R-Ab levels. Spontaneous remission was recorded more often in patients being PLA2R-Ab negative [34]. Analysis of a Dutch cohort $(n=77)$ revealed a correlation of PLA2R$\mathrm{Ab}$ titers with baseline proteinuria, serum creatinine, and baseline estimated glomerular filtration rate (eGFR) after adjustment for fractional IgG excretion (as representative of urinary loss of antibodies). Dividing patients into tertiles based on ELISA titers, renal failure tended to occur more frequently in patients belonging to the group in the highest tertile (PLA2R-Ab titer $>610 \mathrm{U} / \mathrm{ml}$ ) and these patients had a significantly lower incidence of spontaneous remission compared to the lowest (PLA2R-Ab titer 41-175 U/ml) tertile (4 versus $38 \%$, resp.). Accordingly, physicians were prompted to initiate immunosuppression more often in patients in the highest tertile compared to both other groups, whereas time to treatment response was significantly shorter in patients within the lowest tertile [17]. A single-center study from the same institution including 18 primary $\mathrm{MN}$ cases highlighted correlation of PLA2R-Ab with proteinuria, serum B2microglobulin ( $\$ 2 \mathrm{~m})$, urinary IgG excretion, urinary $\$ 2 \mathrm{~m}$, and serum creatinine, whereas eGFR was negatively correlated [19]. Another large study including 118 PLA2R-Ab positive patients established different tertile values, with tertile 1 ranging from 20 to $86 \mathrm{U} / \mathrm{ml}$, tertile 2 from 87 to $201 \mathrm{U} / \mathrm{ml}$, and tertile $3>202 \mathrm{U} / \mathrm{ml}$. Patients in tertile 3 were older, had a higher baseline systolic blood pressure, and tended to have more severe proteinuria. The proportion of patients reaching the predefined primary end point, namely, serum creatinine increase $\geq 25 \%$ and a creatinine value $\geq 1.3 \mathrm{mg} / \mathrm{dl}$, was significantly higher in tertile 3 compared to tertile 1 (69\% versus $25 \%$ ). At the end of follow-up, proteinuria and PLA2RAb levels were higher in patients belonging to tertile 3 [35]. In a cross-sectional analysis, risk of progressive kidney function decline over a long observation period (up to 5 years) was increased in patients with higher PLA2R-Ab levels (high titer $>88 \mu / \mathrm{ml}$ versus low titer $<24 \mu / \mathrm{ml}$ ) [36]. In the GEMRITUX study, a titer of $<275 \mathrm{U} / \mathrm{ml}$ was significantly associated with achieving the primary end point (complete or partial remission) [38]. A clear association between proteinuria 
and serum PLA2R-Ab levels and serum creatinine could be confirmed by a prospective study including 133 patients with detectable antibodies. PLA2R-Ab titers at baseline were significantly higher in those patients who did not experience remission during follow-up. Patients having lower PLA2R$\mathrm{Ab}$ levels reached remission significantly faster compared to those with higher titers at baseline [20]. Another study from India observed a significantly higher proportion of complete remissions in the combined lower and intermediate range PLA2R-Ab tertile compared to those belonging to the highest tertile. Furthermore, resistant disease was common in patients with a baseline titer $>300 \mathrm{RU} / \mathrm{ml}(55.2 \%)$, whereas remission was frequently observed in those with a titer of $<$ $100 \mathrm{RU} / \mathrm{ml}$ at baseline (68.2\%). In general, detectable PLA2RAbs were associated with treatment resistance compared to patients with no antibodies [22]. In a large study from China, detectable PLA2R-Abs $(n=392)$ were associated with a higher level of proteinuria and a lower level of eGFR. A weak correlation of antibody levels with proteinuria, serum albumin, and eGFR was shown. Moreover, patients with a positive PLA2R-Ab test and glomerular PLA2R staining were more likely not to achieve remission compared to those with undetectable antibodies and positive glomerular staining. In general, patients with a higher PLA2R-Ab or glomerular PLA2R rate had a higher proportion of failure to achieve remission. Combined positivity was a significant predictive value for no remission [25]. In line with several other reports, PLA2R-Ab positivity in Korean patients was associated with more severe proteinuria and lower serum albumin [21]. Proteinuria and number of patients having nephrotic range proteinuria were significantly higher in PLA2R-Ab positive cases in a Chinese study [23]. In a Japanese analysis including 19 patients with and without detectable PLA2R-Ab in each group, serum IgG was significantly lower in patients with antibodies, whereas proteinuria tended to be higher [28]. Respective associations/correlations have been highlighted in Table 3.

Taken together, based on the available data it is not possible to provide definite PLA2R-Ab cut-off, since different values have been reported in the respective studies. Nevertheless, in several studies, patients within the highest tertile had worse renal outcome and more frequently treatment resistance towards initiated immunosuppression. Notably, two reports have used purified protein to coat ELISA plates [17, 36], whereas the remainder used a commercially available kit $[22,35,38]$. High PLA2R-Ab titers (exceeding 250-300 IU/L) have been associated with more severe proteinuria, higher serum creatinine/lower eGFR, serum/urinary $ß 2 \mathrm{M}$, and urinary IgG excretion.

\subsection{PLA2R-Ab Presence and Decline Predict Treatment} Response. PLA2R-Abs were present during clinical disease activity, whereas a decline or complete disappearance of PLA2R-Ab preceded resolution of proteinuria [15]. In a first multicenter retrospective study, the effects of rituximab on PLA2R-Ab levels were tested. 25 of 35 (71\%) patients had detectable PLA2R-Ab and these antibodies disappeared or declined in 17 (68\%) patients within 12 months following rituximab treatment, accompanied by a higher rate of complete or partial remission compared to those with a persistent antibody titer (59\% and $88 \%$ versus $0 \%$ and $33 \%$ after 12 and 24 months). Changes in antibody titers preceded changes in proteinuria [40].

An observational study assigning patients either to oral cyclophosphamide or to mycophenolate mofetil and corticosteroids for 12 months evaluated PLA2R-Ab $(n=34 / 48)$ in retrospective. By 2 months of treatment, PLA2R-Ab levels declined from $428 \mathrm{U} / \mathrm{ml}$ to $24 \mathrm{U} / \mathrm{ml}$. Antibody status at the end of treatment predicted 5-year outcome, with 58\% (14/24) of patients with absent PLA2R-Ab achieving persistent remission compared to 0 out of 9 in those with persisting antibodies [41]. Combined treatment with rituximab and cyclosporine for 6 months of revealed PLA2R-Ab negativity in 77.8\% after 3 months and in all at least once during follow-up, which was accompanied by remission. In two patients with relapse, reemergence of PLA2R-Ab was associated with re-occurrence of proteinuria [52]. A recent randomized controlled trial comparing NIAT and rituximab against NIAT alone found significantly more PLA2R-Ab negativity and decreasing titers in the NIAT-rituximab group. In the group with immunologic remission, the primary end point (complete or partial remission after 6 months) was achieved in $43 \%$ compared to $18 \%$ with persistent antibody positivity [38]. In 81 antibodypositive patients receiving a B-cell driven $\left(375 \mathrm{mg} / \mathrm{m}^{2}\right.$ once and a second infusion when more than five circulating Bcells $/ \mathrm{mm} 2$ were detected after one week) or a fixed regimen $\left(375 \mathrm{mg} / \mathrm{m}^{2}\right.$ four times, one week apart), a lower PLA2R$\mathrm{Ab}$ titer and full depletion after six months significantly predicted remission. In general, antibody decline preceded proteinuria and, particularly, a 50\% reduction of PLA2R$\mathrm{Ab}$ preceded equivalent proteinuria reduction by 10 months. Reappearance of PLA2R-Ab predicted disease relapse [37]. A similar pattern of PLA2R-Ab course was found in a Dutch study, showing a significant decline in the remission phase and a reappearance of PLA2R-Ab during relapse [19]. In a prospective German study, a decrease in PLA2R-Ab levels was accompanied by a steady increase in serum albumin. After three months of follow-up, a 45\% decrease of PLA2R-Ab titers was paralleled by a $25 \%$ decrease in proteinuria in a cohort of 133 patients. The changes were even more pronounced in patients receiving immunosuppression, with a decline in PLA2R-Ab levels ranging from 69 to $81 \%$ and proteinuria by $38.8 \%$ [20]. In another analysis from India including 114 patients, a decline over 90\% in PLA2R-Ab decline in PLA2RAb titers by 12 months was accompanied by remission in $85.4 \%$, whereas a decline lower than $50 \%$ in the same period was associated with persistent nephrotic range proteinuria in $87.5 \%$. A correlation between proteinuria and serum albumin with PLA2R-Ab titers at 6 and 12 months was found [22]. In a recent Chinese study comparing PLA2R-related MN $(n=91)$ with negative patients $(n=13)$, the total remission rate was significantly higher in patients with absent PLA2R-Ag staining [39].

A subanalysis of the prospective study conducted by Hoxha and colleagues analyzed the clinical outcome of included patients with nonnephrotic range proteinuria. During the follow-up period, significantly more patients with detectable PLA2R-Ab developed nephrotic range proteinuria 


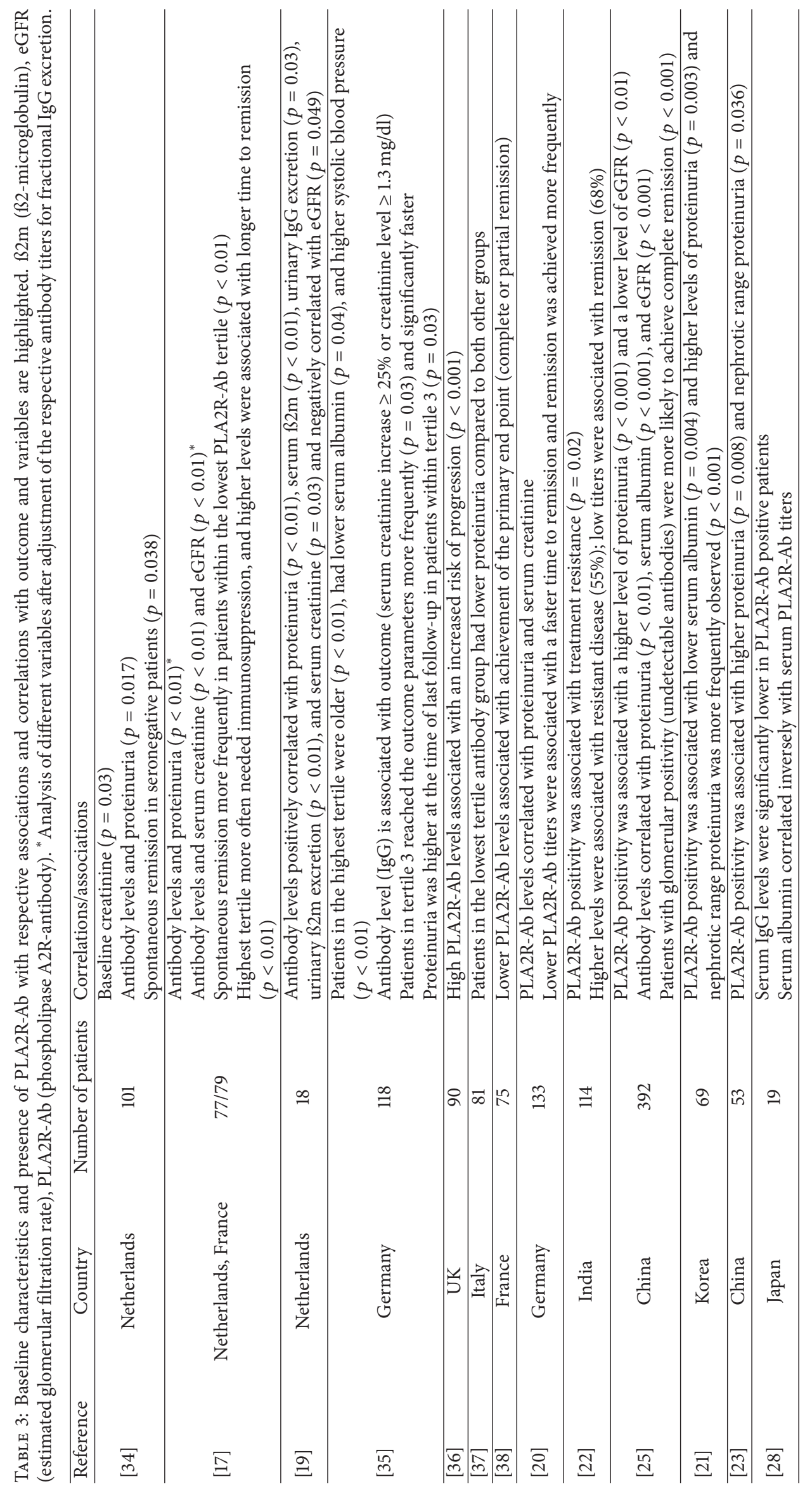


(13 out of 16 patients) compared to those that tested negative (five out of 17). In accordance with this finding, immunosuppression was initiated more often in patients with PLA2R$\mathrm{Ab}$ (13 versus 2 patients, resp.). At the end of follow-up, persistence of PLA2R-Ab was associated with no remission of proteinuria and a decline in renal function [42]. Findings have been highlighted in Table 4 .

Taken together, higher baseline PLA2R-Ab titers are associated with worse renal outcome and resistance towards immunosuppressive therapy. In general, decline in antibody levels precede changes in proteinuria by months, irrespective of the immunosuppressive measure. Moreover, reappearance of PLA2R-Ab is a strong predictor of disease relapse.

\subsection{Recurrence of Disease after Transplantation and PLA2R-} $A b$. In a study by Quintana and colleagues, recurrence was observed in 7 of 21 (33.3\%) patients. PLA2R-Abs were measured ahead of kidney transplantation and were positive in $85.7 \%$ and $35.7 \%$ of the recipients with and without recurrence, respectively. A correlation of PLA2R-Ab positivity at the time of graft biopsy and before transplantation with recurrence of primary $\mathrm{MN}$ was found by the authors. A PLA2R-Ab cut-off level of $45 \mathrm{U} / \mathrm{ml}$ could predict recurrence, with a sensitivity of $85.3 \%$ and a specificity of $85.1 \%$. Presence of HLA-DQA1 05:01(05) and HLA-DQB1 02:01 was reported in 6 of 7 patients with recurrent $M N$ and was associated with the highest pretransplantation PLA2R-Ab levels [53]. These findings were in part corroborated by another singlecenter study. Among the six patients (37.5\%) with recurrence, a positive ELISA with a titer $>30 \mathrm{RU} / \mathrm{ml}$ provided a sensitivity and specificity of $83 \%$ and $100 \%$, respectively. The risk alleles were found in 5 out of six patients $(83 \%)$. Combination of both above-mentioned studies $(n=37)$ yielded a sensitivity of $85 \%$ and a specificity of $92 \%$ to predict recurrence with a PLA2R-Ab titer of $>29 \mathrm{U} / \mathrm{ml}$ [54]. Another study including 10 patients with primary $\mathrm{MN}$ reported an association of persistent IgG4 PLA2R-Ab and the respective antibody titer after six months with the recurrence of disease [55]. Moreover, persistence or reappearance of PLA2R-Ab after kidney transplantation was associated with increasing proteinuria and treatment-resistant recurrent $\mathrm{MN}$ in another case series [56]. Taken together, a PLA2R-Ab titer before renal transplantation $>30$ or $>45 \mathrm{U} / \mathrm{ml}$ (depending on the respective publications), persistence, or reappearance during follow-up may predict recurrence of disease. This needs to be confirmed by further studies assessing patients in a prospective manner.

\subsection{Immunodominant Epitope Region and Impact of Epitope} Spreading on Outcome. Epitopes were identified by SPOT (peptide arrays on membrane) technology and detected by Western blot. Restricting analysis to the IgG4 subclass yielded lower background and stronger signals localized to seven consensus epitopes, which were in the extracellular domain of the PLA2R. Six of them were localized to the C-type lectin-like domains (CTLDs, one in CTLD1, one in CTLD2, two in CTLD6, and two in CTLD8), whereas one was located to the $\mathrm{N}$-terminal cysteine-rich region (C-R)
[57]. Further research aimed at deciphering the responsible epitope responsible for PLA2R-Ab binding. Fresquet et al. identified the major epitope within the N-terminus to CTLD3 (comprising a complex of CysR, FnII, and CTLD1-CTLD 3) of PLA2R which was recognized by $90 \%$ of human PLA2R-Ab and effectively competed for the binding with fulllength PLA2R. Further experiments using mass spectrometry revealed a pivotal role of peptide 1 and peptide 2 , representing the $B 1$ and $B 3$ strain from the ricin domain. Extension of peptide 1 towards peptide 2 is exerted by a 31-mer within the cysteine-rich (CysR) region, and competitive binding analyses revealed the essential role of 31-mer in the binding of PLA2R-Ab [58]. In line with the results described above, Kao and colleagues reported recognition of patient sera by a protein complex consisting of the CysR, fibronectin-like type II (FnII), and CTLD1 domains of PLA2R. Binding analysis comparing full-length PLA2R and the complex of CysR-FnIICTLD1 indicated an equally efficacy in the recognition of PLA2R-Ab positive patient sera [59]. Since $10 \%$ of patients did not show binding to the described epitopes, it was assumed that a further epitope in the more C-terminal region may exist [60]. Further in depth analyses led to the identification of the three pivotal epitopes responsible for PLA2R-Ab binding. Seitz-Polski et al. described the loss of recognition in all tested patients $(n=50)$ after the deletion of the three epitopes, namely CysR, CTLD1 and CTLD7. Based on their findings, they set up ELISAs to specifically measure presence of antibodies directed against these domains. A total of 69 patients with presence of PLA2R were tested, and CysR as the dominant and most common epitope was recognized in 68 patients, whereas the CTLD1 and CTLD7 domains were recognized in 42 and 32 sera, respectively. Based on these findings, they classified patients according to antibody presence into a CysR (CysR antibodies only, $n=23$ ), CysRC1 (CysR and CTLD1 only, $n=14$ ) and a CysRC1C7 group (with presence of antibodies against all domains, $n=32$ ). The CysR antibodies were significantly higher in the latter two groups compared to the CysR group. Patients in the CysR group were significantly younger and their baseline proteinuria was lower compared to the other two groups, while a proteinuria over $5 \mathrm{~g} / \mathrm{g}$ creatinine was most frequently found in the CysRC1C7 group. Furthermore, a progressive increase of proteinuria was accompanied by a progressive increase in CTLD7. Assessment of outcome revealed significantly more spontaneous remissions in the CysR group, whereas the occurrence of end stage renal increase was limited to the CysRC1 and CysRC1C7 groups and proteinuria increased from CysR to CysRC1C7. In accordance, the proportion of patients with a $30 \%$ increase in serum creatinine was higher in the CysRC1 and CysRC1C7 groups. Multivariate analysis retained CysRC1, CysRC1C7 and high titers of PLA2R-Ab as independent risk factors for poor renal prognosis. Serial samples were available for a subset of patients and an epitope switch was observed in a minority, associated with a change in clinical presentation (i.e., one patients switched from the CysRC1C7 to the CysR group and entered remission following initiation of immunosuppression). ELISA tests were set up for the detection of IgG4 antibodies only. Analysis 


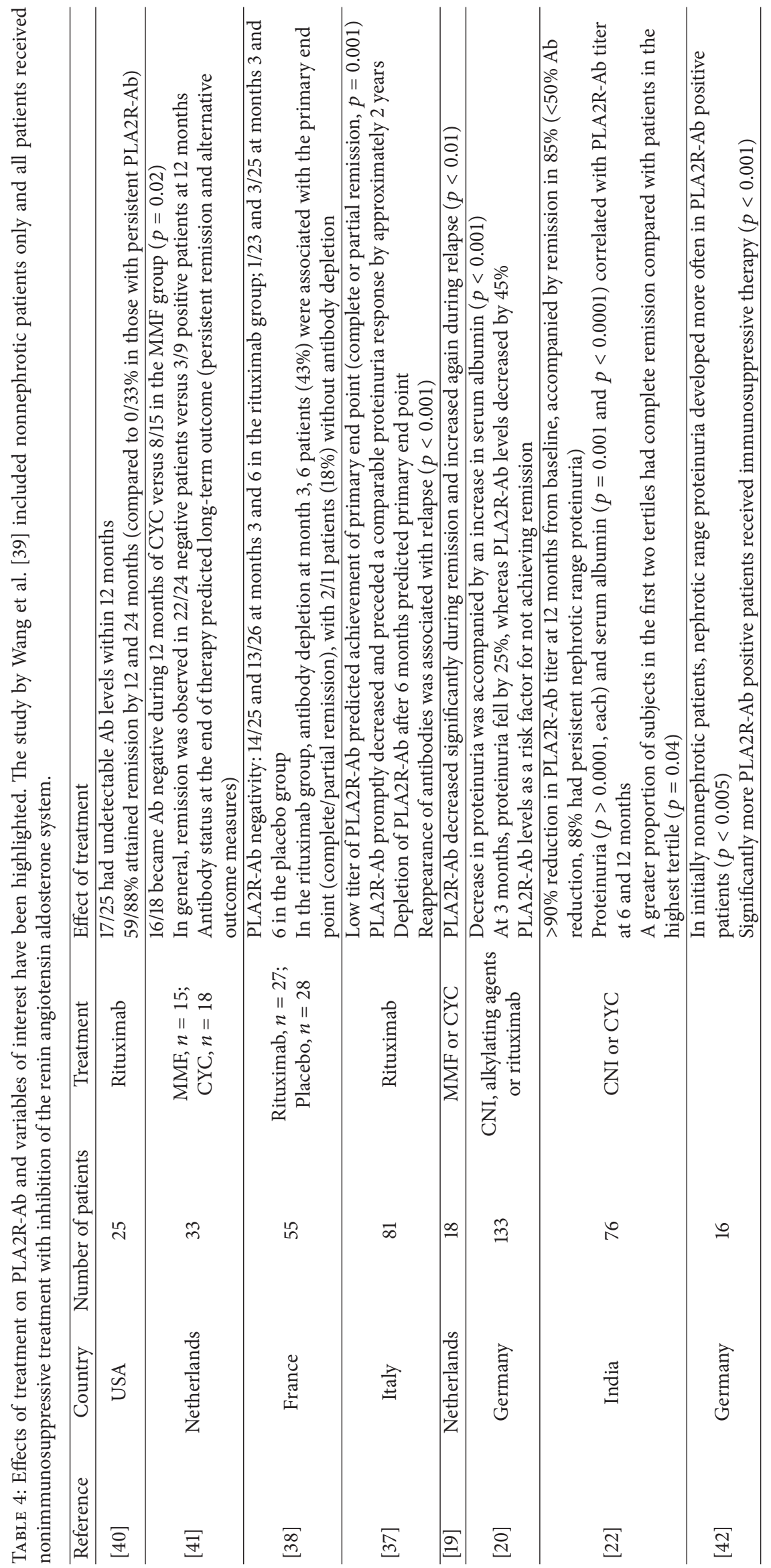


of other subclasses highlighted that anti-CTLD7 antibodies were exclusively restricted to the IgG4 subclass [61].

The identification of the immunodominant epitope regions of PLA2R have further provided insight into pathogenesis. Moreover, the CysRC1, CysRC1C7 and higher titers of circulating PLA2R-Ab may be considered as independent risk factors for poor prognosis. Moreover, epitope spreading has been observed, indicating changes in both directions (high risk phenotype to the CysR group and vice versa). It is tempting to speculate that specific developments (i.e., commercially available ELISA kits) will further help to facilitate identification of patients bearing a high risk of treatment resistance or progressing to end stage renal failure.

2.6. Identification of Susceptible Loci Associated with Primary Membranous Nephropathy. A genome-wide association study (GWAS) was employed to identify risk alleles responsible for the onset of primary MN. Three cohorts (with Dutch, French and British patients) were analyzed and independently displayed a significant association with HLA$D Q A 1$ on chromosome $6 \mathrm{p} 21$. In a joint analysis, comprising 556 patients with primary $\mathrm{MN}$ and 2338 controls, the authors identified the most significant association within HLA-DQA1 and PLA2R1 on chromosome 2q24 [62]. A subsequent study from Spain including 89 primary $\mathrm{MN}$ patients and 286 controls confirmed genetic associations with $H L A-D Q A 1$ and PLA2R1. Interestingly, combination of the susceptibility genotypes (A/A and A/G for HLA-DQA1 and A/A for PLA2R1) significantly predicted response to treatment, which became even more apparent after adjustment for baseline proteinuria. Moreover, carriers of the $\mathrm{A} / \mathrm{A}$ or $\mathrm{A} / \mathrm{G}$ genotype for $H L A-D Q A 1$ had a longer mean doubling of serum creatinine-free survival [63]. A study from Germany observed an association between classical HLA alleles (DRB1 ${ }^{*} 0301-D Q A 1^{*} 0501-D Q B^{*} 0201$ haplotype) and primary $M N$. Whereas $P L A 2 R 1$ was exclusively identified in primary $\mathrm{MN}$, the $H L A-D Q A 1$ risk allele was associated with LN, diabetic nephropathy and FSGS as well [64]. In a crosssectional study from the UK, HLA-DQA1* 05:01 and $H L A-$ $D Q B 1^{*} 02.01$ were associated with higher PLA2R-Ab levels [36]. In a large monocentric study, analysis of the risk alleles revealed that homozygous carriage of the PLA2R1 A allele was associated with a 2-fold higher PLA2R-Ab level compared to $\mathrm{A} / \mathrm{G}$ and $\mathrm{G} / \mathrm{G}$ carriers [37].

Similar results were reported in non-Caucasian patients with primary MN. A study from India compared 114 cases and 95 healthy controls and observed differences in the PLA2R1 and HLA-DQA1 SNPs which became more evident when studying PLA2R-related cases only [22]. In a large Northern American analysis, PLA2R1 SNPs were associated with PLA2R-related $\mathrm{MN}$ in Caucasians only. A strong epistasis between PLA2R1 and HLA-DQA1 was found [65]. In African Americans, 120 PLA2R-related MN biopsies were screened for the presence of APOL1 risk alleles, identifying 46 cases with 0,51 cases with 1 and 23 cases with 2 APOL1 risk alleles. Presence of two risk alleles was associated with histologic features implicated in worse renal outcome [66].

Analysis of 1112 Chinese patients and 1020 healthy controls revealed a strong association between three SNPs within
PLA2R1 and one SNP within HLA-DQA1 and a potentiation when risk alleles were combined [67]. Restricting analysis to the MHC region in PLA2R-positive primary MN, HLA$D R B 1^{*} 15: 01$ and $H L A-D R B 3^{*} 02: 02$ were strongly associated. Whereas HLA-DRB3* 02:02 was found in a comparable number of PLA2R-negative MN, HLA-DRB1*15:01 was found in $81.8 \%$ of patients with PLA2R-related MN compared to $16 \%$ of PLA2R-negative MN and $21 \%$ of the healthy controls. A replication cohort confirmed the findings of both HLA loci. Interestingly, in a joint analysis, 387 patients $(98.7 \%)$ carried at least one of the proposed HLA risk alleles [68]. Another Chinese study included 261 cases and 599 healthy controls. $H L A-D R B 1^{*} 15: 01$ and $H L A-D R B 1^{*}$ 03:01 were independently associated with primary MN. PLA2R-Ab positivity was associated with the carriage of one of the risk alleles, whereas association remained at a lower degree for $D R B 1^{*} 15: 01$, while $D R B 1^{*}$ 03:01 lost its effect [69]. In a Japanese cohort comprising 183 patients with primary $\mathrm{MN}$ and 811 healthy controls, several tested PLA2R1 SNPs were associated. Moreover, the association of $H L A-D R B 1^{*} 15: 01$ could also be observed, whereas $H L A-D Q B 1^{*}$ 06:02 emerged as a second independent risk allele [70]. To identify rare genetic variants in $P L A 2 R 1$, all 30 exons of PLA2R1 were sequenced. Although the general association between PLA2R1 and primary $M N$ could be confirmed, no evidence was provided that rare variants within the coding region cause the proposed association between primary $\mathrm{MN}$ and the PLA2R1 gene [71].

Despite progress in the understanding of genetics related to primary $\mathrm{MN}$, there is a lack of heritability. One report from 1984 highlighted occurrence of MN in three pairs of brothers, with one pair being monozygotic (HLA-identical) twins [72]. In general, the presence of HLA-DQA1 and PLA2R1 risk alleles confers a significant association with primary $\mathrm{MN}$ and the combination of risk phenotypes potentiates the risk to develop MN in most ethnicities. Particularly, MHC class II molecules play a pivotal role in $\mathrm{MN}$. In Chinese patients with primary $\mathrm{MN}$, carriage of $H L A-D R B 1^{*} 15: 01$ confers a significant risk to develop PLA2R-related MN, whereas HLA$D R B 1^{*}$ 03:01 or $H L A-D R B^{*}$ 06:02 may carry a risk to develop either PLA2R-related or unrelated $\mathrm{MN}$.

\section{Thrombospondin Type 1 Domain-Containing 7A in (Primary) Membranous Nephropathy}

Since about $20-30 \%$ of cases with primary MN may exhibit other antigens, a similar approach as with the discovery of PLA2R-Ab was chosen to identify further antigens. Thrombospondin type 1 domain-containing 7A (THSD7A) was identified by Western-blotting and subsequent mass spectrometry and has a molecular size of approximately $250 \mathrm{kDa}$. Among patients with PLA2R-Ab negative primary $\mathrm{MN}$, THSD7A was found in 6 out of 44 patients in a European and in 9 out of 110 patients in a US cohort. In accordance with PLA2R-Ab, IgG4 was the predominant IgG subclass of anti-THSD7A, and colocalization of IgG4 and THSD7A was observed in renal tissue specimens. Staining for nephrin colocalized with staining for THSD7A, suggesting expression 
of THSD7A in proximity to the podocyte foot processes. Interestingly, anti-THSD7A antibodies were more frequently observed in women (65\% versus 27\%) [16]. A single-center study from Japan including consecutive patients with $\mathrm{MN}$ found THSD7A-related MN in five out of 55 patients (9.1\%). Again, total numbers were low, but the authors found that patients with THSD7A-related MN were significantly younger compared to PLA2R-related $\mathrm{MN}$ and a female predominance (three out of five), which was in contrast to patients being PLA2R-positive [29]. Lower prevalence was reported in a Chinese cohort (three patients with primary $\mathrm{MN}$ ), and additionally two patients had secondary causes (one tumor-associated and one hepatitis B-associated, overall $3.7 \%)$. Importantly, IgG4 staining was positive in primary and secondary causes [73]. Analysis of consecutive biopsies from the Boston cohort revealed a positive staining for THSD7A in 7/258 (3\%) patients, whereas two cases presented with dual (PLA2R and THSD7A) positivity (1\%) [74]. Concomitant occurrence of THSD7A-related MN and mixed adenoneuroendocrine carcinoma of the gallbladder with positive staining in the kidney, the gallbladder and an affected lymph node highlighted a potential link between THSD7Apositivity and malignancy. Screening of 1009 patients with MN revealed further 25 patients with positive anti-THSD7A antibodies. Of these, seven had a malignancy [75]. Further analysis of consecutive MN patients revealed a prevalence of $2.6 \%$ out of 345 patients, with a majority being female. The association between THSD7A-related MN and malignant disease exceeded the number of PLA2R-related MN and malignancy. After inclusion of the retrospective cohorts and analysis of samples derived from $1276 \mathrm{MN}$ patients, 8 out of 40 patients with detectable anti-THSD7A antibodies presented with malignancy and the median time from diagnosis of $\mathrm{MN}$ and development or detection of malignancy was 3 months. Again, a female predominance was reported by the authors. Achievement of complete remission was accompanied by undetectable anti-THSD7A antibody titers, whereas a majority of those with partial remission and one patient with no remission remained positive [76]. In contrast to PLA2R1, THSD7A is expressed on both murine and human podocytes, and administration of human anti-THSD7A antibodies bind to murine THSD7A, leading to proteinuria and a histopathology resembling MN [77]. This finding confirmed the assumption that antigen-antibody interaction is pivotal in the onset of MN.

Taken together, anti-THSD7A antibodies are a novel antigen which is more frequently present in Japanese patients with primary MN. In particular, research needs to decipher its association with malignancy and intensive screening may be warranted in positive patients, since the number of observed malignant diseases may be underestimated by the current reports due to a short follow-up period.

\section{Rare Forms of Childhood Membranous Nephropathy and Potential Ways to the Detection of Novel Antigens}

$\mathrm{MN}$ is a common cause of nephrotic syndrome in adults; it is rare, however in children $[78,79]$. Since PLA2R and THSD7A do not seem to play any major role in infants and young children, other auto- or allo-antigens must looked for, after secondary causes have been excluded thoroughly, particularly malignancy. Recently, rare causes of $\mathrm{MN}$ were identified in children, such as antibodies to neutral endopetidase (NEP).

A case of antenatal MN with a severe, unusual histopathological presentation was further investigated, since the transplacental passage of nephritogenic antibodies was suspected to be causative. Sequential serum samples from the mother (before and after delivery) and serum of the infant after birth showed reactivity with glomerular capillary walls and the brush border. A $90 \mathrm{kDa}$ antigen was found, and further experiments identified NEP as the antigenic fraction. Kidney biopsy of the infant and of rabbits after incubation with maternal IgG revealed colocalization of IgG antibodies and NEP in subepithelial immune deposits. The mother's granulocytes exhibited a lack of NEP expression, thus an alloimmunization process was discussed as responsible pathogenetic step [80]. Truncating mutations in the metallomembrane endopeptidase ( $M M E$ ) gene were found to be causative of alloimmunization during pregnancy, leading to the production of anti-NEP antibodies. Whereas infants of four mothers with mutations had oligoanuria, nephrotic syndrome or renal failure, all children of one mother had no overt renal disease. Analysis of anti-NEP antibodies revealed an approximately 10-fold lower titer for IgG as compared to those of the remaining mothers. Rapid improvement of kidney failure and nephrotic syndrome was observed in affected infants [14]. Two mothers were followed during their second pregnancy. One mother developed a marked increase in anti-NEP IgG1 and IgG4 antibodies. Severe disease occurred despite treatment strategies aiming at reducing its levels. The other mother produced anti-NEP IgG4 antibodies exclusively and delivery of a healthy newborn was possible [81]. More recently, two additional mothers (from Germany and Italy) bearing a truncating deletion in the $M M E$ gene were identified. The German mother produced predominantly anti-NEP IgG4 antibodies, whereas the Italian mother mainly produced complement-fixing anti-NEP IgG1 antibodies, the latter able to inhibit NEP enzymatic activity. Whereas the German child had rapid regression of all symptoms, the Italian child required treatment with intermittent peritoneal dialysis and an impaired renal function at the time of last follow-up was reported. These findings highlight that complement-fixing anti-NEP IgG1 antibodies are necessary to develop a severe disease phenotype [82].

Various reports have already indicated a role for allergens, including dietary allergens in the pathogenesis of renal glomerular diseases. Early studies demonstrated that some patients develop relapses after cow's milk exposure and elimination of milk from the diet could prevent relapses of lipoid nephrosis [83]. This has been explained by a dysregulation of the IL-13 system [84], leading to overproduction of IgE-specific antibodies of food compounds, including cow's milk and theoretically also bovine serum albumin (BSA). Anti-BSA antibodies, commonly prevalent in the general population, have been detected in high levels in 4 of 5 consecutive children (age $<5$ years) and 7 of 41 consecutive adults with MN. Elevated levels of circulating BSA were 
also present in those children with high levels of anti-BSA antibodies. As shown in various animal models, the cationic form of BSA is able to induce MN. Subepithelial deposits of BSA were detected in children with both, high levels of circulating cationic BSA and antibodies, and in the absence of PLA2R [85].

Gel electrophoresis identified a few proteins which were repetitively recognized by $\mathrm{MN}$ sera. Among these, antibodies against aldose reductase (AR), manganese superoxide dismutase (SOD2) were elevated in MN compared to FGSG and normal controls. Analysis of kidney specimen revealed marked expression of AR and SOD2 in glomeruli of patients with $\mathrm{MN}$, and colocalization of both, AR and SOD2, with IgG4 and the terminal complement complex [86]. A proteomic approach found three immune proteins in glomeruli of patients with $\mathrm{MN}$, namely $\alpha$-enolase ( $\alpha \mathrm{ENO})$, elongation factor 2 and glycyl aminoacyl-tRNA synthetase. One of the factors, $\alpha \mathrm{ENO}$, fulfilled the criteria and was considered as autoantigen, colocalized with IgG4 in deposits, and was found to be elevated in $25 \%$ of $131 \mathrm{MN}$ patients [87]. Moreover, PLA2R Ab levels correlated with antibodies against AR, SOD2 and $\alpha$ ENO [88]. Recently, Kimura found no difference in the frequency of $\alpha \mathrm{ENO}$ antibodies between primary and secondary $\mathrm{MN}$, although IgG4 antibodies were exclusively found in primary MN [89].

Taken together, rare antigens have been observed in antenatal or early-onset membranous nephropathy. In cases with anti-NEP antibodies, preponderance of IgG4 antibodies was associated with a good prognosis, whereas a predominance of IgG1 was accompanied by a severe disease phenotype. Moreover, a role for the cationic form of BSA to cause membranous nephropathy in children has been described and circulating antibodies have been observed. Rarer antigens have been discovered by gel electrophoresis. One can speculate that more antigens will be discovered in near future.

\section{Conclusions and Outlook}

The aim of the present review was to summarize recent literature related to the immunology of primary $\mathrm{MN}$. While PLA2R-Ab represents the leading circulatory antibody in primary $\mathrm{MN}$ with a presence of $70-80 \%$, the role of antibodies to THSD7A needs to be better defined, since malignancyassociated THSD7A-related MN has recently been reported to occur in a significant proportion of patients. Other antibodies leading to secondary forms of $\mathrm{MN}$ have not been described so far, and there is still room for further antibody discovery. Moreover, primary MN has a clear genetic basis, but reports related to genetic heritability are widely lacking. In addition, novel ELISA kits detecting different epitopes of PLA2R-Ab may pave the way to distinguish PLA2Rassociated MN patients with a predictable poor and favorable outcome. Rare antibodies have been identified in childhood or antenatal MN. With this increasing research in mind, it is likely that we will learn more about the etiopathogenesis of primary and secondary $\mathrm{MN}$ in near future.

\section{Conflicts of Interest}

The authors declare that they have no conflicts of interest.

\section{References}

[1] C. Ponticelli and R. J. Glassock, "Glomerular diseases: membranous nephropathy-a modern view," Clinical Journal of the American Society of Nephrology, vol. 9, no. 3, pp. 609-616, 2014.

[2] T. Alfaadhel and D. Cattran, "Management of Membranous Nephropathy in Western Countries," Kidney Diseases, vol. 1, no. 2, pp. 126-137, 2015.

[3] C. C. Huang, A. Lehman, A. Albawardi et al., "IgG subclass staining in renal biopsies with membranous glomerulonephritis indicates subclass switch during disease progression," Modern Pathology, vol. 26, no. 6, pp. 799-805, 2013.

[4] A. M. Davies and B. J. Sutton, "Human IgG4: A structural perspective," Immunological Reviews, vol. 268, no. 1, pp. 139-159, 2015.

[5] J. H. Stone, Y. Zen, and V. Deshpande, "Mechanisms of disease: IgG4-related disease," The New England Journal of Medicine, vol. 366, no. 6, pp. 539-551, 2012.

[6] T. Kamisawa, Y. Zen, S. Pillai, and J. H. Stone, "IgG4-related disease," The Lancet, 2014.

[7] A. McGrogan, C. F. M. Franssen, and C. S. De Vries, "The incidence of primary glomerulonephritis worldwide: A systematic review of the literature," Nephrology Dialysis Transplantation, vol. 26, no. 2, pp. 414-430, 2011.

[8] D. C. Cattran, H. N. Reich, H. J. Beanlands, J. A. Miller, J. W. Scholey, and S. Troyanov, "The impact of sex in primary glomerulonephritis," Nephrology Dialysis Transplantation, vol. 23, no. 7, pp. 2247-2253, 2008.

[9] O. Moranne, L. Watier, J. Rossert, and B. Stengel, "Primary glomerulonephritis: An update on renal survival and determinants of progression,” QJM, vol. 101, no. 3, pp. 215-224, 2008.

[10] Y.-H. Chou, Y.-C. Lien, F.-C. Hu et al., "Clinical outcomes and predictors for ESRD and mortality in primary GN," Clinical Journal of the American Society of Nephrology, vol. 7, no. 9, pp. 1401-1408, 2012.

[11] J. J. Sim, M. Batech, A. Hever et al., "Distribution of BiopsyProven Presumed Primary Glomerulonephropathies in 20002011 Among a Racially and Ethnically Diverse US Population," American Journal of Kidney Diseases, vol. 68, no. 4, pp. 533-544, 2016.

[12] A. Kronbichler, M. A. Saleem, B. Meijers, and J. I. Shin, "Soluble Urokinase Receptors in Focal Segmental Glomerulosclerosis: A Review on the Scientific Point of View," Journal of Immunology Research, vol. 2016, Article ID 2068691, 2016.

[13] G. Moroni, B. Gallelli, S. Quaglini et al., "Long-term outcome of renal transplantation in patients with idiopathic membranous glomerulonephritis (MN)," Nephrology Dialysis Transplantation, vol. 25, no. 10, pp. 3408-3415, 2010.

[14] H. Debiec, J. Nauta, F. Coulet et al., "Role of truncating mutations in MME gene in fetomaternal alloimmunisation and antenatal glomerulopathies," Lancet, vol. 364, no. 9441, pp. $1252-1259,2004$.

[15] L. H. Beck Jr., R. G. B. Bonegio, G. Lambeau et al., "M-type phospholipase A2 receptor as target antigen in idiopathic membranous nephropathy," The New England Journal of Medicine, vol. 361, no. 1, pp. 11-21, 2009.

[16] N. M. Tomas, L. H. Beck Jr., C. Meyer-Schwesinger et al., "Thrombospondin type-1 domain-containing 7A in idiopathic membranous nephropathy," The New England Journal of Medicine, vol. 371, no. 24, pp. 2277-2287, 2014.

[17] J. M. Hofstra, H. Debiec, C. D. Short et al., "Antiphospholipase $A_{2}$ receptor antibody titer and subclass in idiopathic 
membranous nephropathy," Journal of the American Society of Nephrology, vol. 23, no. 10, pp. 1735-1743, 2012.

[18] E. Hoxha, U. Kneiler, G. Stege et al., "Enhanced expression of the M-type phospholipase A2 receptor in glomeruli correlates with serum receptor antibodies in primary membranous nephropathy," Kidney International, vol. 82, no. 7, pp. 797-804, 2012.

[19] J. M. Hofstra, L. H. Beck Jr., D. M. Beck, J. F. Wetzels, and D. J. Salant, "Anti-phospholipase $\mathrm{A}_{2}$ receptor antibodies correlate with clinical status in idiopathic membranous nephropathy," Clinical Journal of the American Society of Nephrology, vol. 6, no. 6, pp. 1286-1291, 2011.

[20] E. Hoxha, I. Thiele, G. Zahner, U. Panzer, S. Harendza, and R. A. K. Stahl, "Phospholipase A2 receptor autoantibodies and clinical outcome in patients with primary membranous nephropathy," Journal of the American Society of Nephrology, vol. 25, no. 6, pp. 1357-1366, 2014.

[21] Y. J. Oh, S. H. Yang, D. K. Kim, S.-W. Kang, and Y. S. Kim, "Autoantibodies against phospholipase $\mathrm{A}_{2}$ receptor in Korean patients with membranous nephropathy," PLoS ONE, vol. 8, no. 4, Article ID e62151, 2013.

[22] R. Ramachandran, V. Kumar, A. Kumar et al., "PLA2R antibodies, glomerular PLA2R deposits and variations in PLA2R1 and HLA-DQA1 genes in primary membranous nephropathy in South Asians," Nephrology Dialysis Transplantation, vol. 31, no. 9, pp. 1486-1493, 2016.

[23] X. Li, D. Wei, Z. Zhou et al., "Anti-PLA2R antibodies in chinese patients with membranous nephropathy," Medical Science Monitor, vol. 22, pp. 1630-1636, 2016.

[24] W. Qin, L. H. Beck Jr., C. Zeng et al., "Anti-phospholipase A2 receptor antibody in membranous nephropathy," Journal of the American Society of Nephrology, vol. 22, no. 6, pp. 1137-1143, 2011.

[25] H. Qin, M. Zhang, W. Le et al., "Combined Assessment of Phospholipase A2 Receptor Autoantibodies and Glomerular Deposits in Membranous Nephropathy," Journal of the American Society of Nephrology, vol. 27, no. 10, pp. 3195-3203, 2016.

[26] H.-R. Dong, Y.-Y. Wang, X.-H. Cheng et al., "Retrospective study of phospholipase A2 receptor and IgG subclasses in glomerular deposits in Chinese patients with membranous nephropathy," PLoS ONE, vol. 11, no. 5, Article ID e0156263, 2016.

[27] S. Akiyama, M. Akiyama, E. Imai, T. Ozaki, S. Matsuo, and S. Maruyama, "Prevalence of anti-phospholipase A2 receptor antibodies in Japanese patients with membranous nephropathy," Clinical and Experimental Nephrology, vol. 19, no. 4, pp. 653660, 2014.

[28] K. Hihara, M. Iyoda, S. Tachibana et al., "Anti-phospholipase A2 receptor (PLA2R) antibody and glomerular PLA2R expression in Japanese patients with membranous nephropathy," PLoS ONE, vol. 11, no. 6, Article ID e0158154, 2016.

[29] T. Iwakura, N. Ohashi, A. Kato, S. Baba, and H. Yasuda, "Prevalence of enhanced granular expression of thrombospondin type-1 domain-containing 7A in the Glomeruli of Japanese patients with idiopathic membranous nephropathy," PLoS ONE, vol. 10, no. 9, Article ID e0138841, 2015.

[30] I. Gunnarsson, W. Schlumberger, and J. Rönnelid, "Antibodies to M-type phospholipase A2 receptor (PLA2R) and membranous lupus nephritis," American Journal of Kidney Diseases, vol. 59, no. 4, pp. 585-586, 2012.

[31] C. P. Larsen, N. C. Messias, F. G. Silva, E. Messias, and P. D. Walker, "Determination of primary versus secondary membranous glomerulopathy utilizing phospholipase A2 receptor staining in renal biopsies," Modern Pathology, vol. 26, no. 5, pp. 709-715, 2013.

[32] Q. Xie, Y. Li, J. Xue et al., "Renal Phospholipase A2 Receptor in Hepatitis B Virus-Associated Membranous Nephropathy," American Journal of Nephrology, vol. 41, no. 4-5, pp. 345-353, 2015.

[33] Y.-C. Ge, B. Jin, C.-H. Zeng et al., "PLA2R antibodies and PLA2R glomerular deposits in psoriasis patients with membranous nephropathy," BMC Nephrology, vol. 17, no. 1, article no. 185,2016

[34] S. A. M. E. G. Timmermans, J. G. M. C. Damoiseaux, P. T. J. Heerings-Rewinkel et al., "Evaluation of anti-PLA2R1 as measured by a novel ELISA in patients with idiopathic membranous nephropathy: A cohort study," American Journal of Clinical Pathology, vol. 142, no. 1, pp. 29-34, 2014.

[35] E. Hoxha, S. Harendza, H. Pinnschmidt, U. Panzer, and R. A. K. Stahl, "M-type phospholipase A2 receptor autoantibodies and renal function in patients with primary membranous nephropathy," Clinical Journal of the American Society of Nephrology, vol. 9, no. 11, pp. 1883-1890, 2014.

[36] D. Kanigicherla, J. Gummadova, E. A. McKenzie et al., "AntiPLA2R antibodies measured by ELISA predict long-term outcome in a prevalent population of patients with idiopathic membranous nephropathy," Kidney International, vol. 83, no. 5, pp. 940-948, 2013.

[37] P. Ruggenenti, H. Debiec, B. Ruggiero et al., "Anti-Phospholipase A2 receptor antibody titer predicts post-rituximab outcome of membranous nephropathy," Journal of the American Society of Nephrology, vol. 26, no. 10, pp. 2545-2558, 2015.

[38] K. Dahan, H. Debiec, E. Plaisier et al., "Rituximab for severe membranous nephropathy: a 6-month trial with extended follow-up," Journal of the American Society of Nephrology, vol. 28, no. 1, pp. 348-358, 2017.

[39] J. Wang, Q. Xie, Z. Sun et al., "Response to immunosuppressive therapy in PLA2R- associated and non-PLA2R- associated idiopathic membranous nephropathy: a retrospective, multicenter cohort study," BMC Nephrology, vol. 18, no. 1, 2017.

[40] L. H. Beck Jr., F. C. Fervenza, D. M. Beck et al., "Rituximabinduced depletion of anti-PLA2R autoantibodies predicts response in membranous nephropathy," Journal of the American Society of Nephrology, vol. 22, no. 8, pp. 1543-1550, 2011.

[41] A. P. Bech, J. M. Hofstra, P. E. Brenchley, and J. F. Wetzels, "Association of anti-PLA 2 R antibodies with outcomes after immunosuppressive therapy in idiopathic membranous nephropathy," Clinical Journal of the American Society of Nephrology, vol. 9, no. 8, pp. 1386-1392, 2014.

[42] E. Hoxha, S. Harendza, H. Pinnschmidt, U. Panzer, and R. A. K. Stahl, "PLA2R antibody levels and clinical outcome in patients with membranous nephropathy and non-nephrotic range proteinuria under treatment with inhibitors of the reninangiotensin system," PLoS ONE, vol. 9, no. 10, Article ID e110681, 2014.

[43] B. Svobodova, E. Honsova, P. Ronco, V. Tesar, and H. Debiec, "Kidney biopsy is a sensitive tool for retrospective diagnosis of PLA2R-related membranous nephropathy," Nephrology Dialysis Transplantation, vol. 28, no. 7, pp. 1839-1844, 2013.

[44] S. Kanda, S. Horita, T. Yanagihara, A. Shimizu, and M. Hattori, "M-type phospholipase A2 receptor (PLA2R) glomerular staining in pediatric idiopathic membranous nephropathy," Pediatric Nephrology, vol. 32, no. 4, pp. 713-717, 2017. 
[45] L. N. Cossey, P. D. Walker, and C. P. Larsen, "Phospholipase A2 receptor staining in pediatric idiopathic membranous glomerulopathy," Pediatric Nephrology, vol. 28, no. 12, pp. 2307-2311, 2013.

[46] V. Kumar, A. Varma, R. Nada et al., "Primary Membranous Nephropathy in adolescence A Prospective study," Nephrology, 2016.

[47] H. Dai, H. Zhang, and Y. He, "Diagnostic accuracy of PLA2R autoantibodies and glomerular staining for the differentiation of idiopathic and secondary membranous nephropathy: An updated meta-analysis," Scientific Reports, vol. 5, article no. 8803, 2015.

[48] A.-E. Van De Logt, J. M. Hofstra, and J. F. M. Wetzels, "Serum anti-PLA2R antibodies can be initially absent in idiopathic membranous nephropathy: Seroconversion after prolonged follow-up," Kidney International, vol. 87, no. 6, pp. 1263-1264, 2015.

[49] M.-J. Guerry, P. Vanhille, P. Ronco, and H. Debiec, "Serum antiPLA2R antibodies may be present before clinical manifestations of membranous nephropathy," Kidney International, vol. 89, no. 6, p. 1399, 2016.

[50] T. Stehlé, V. Audard, P. Ronco, and H. Debiec, "Phospholipase A2 receptor and sarcoidosis-associated membranous nephropathy," Nephrology Dialysis Transplantation, vol. 30, no. 6, pp. 1047-1050, 2015.

[51] A. S. De Vriese, R. J. Glassock, K. A. Nath, S. Sethi, and F. C. Fervenza, "A Proposal for a serology-based approach to membranous nephropathy," Journal of the American Society of Nephrology, vol. 28, no. 2, pp. 421-430, 2017.

[52] M. Waldman, L. H. Beck, M. Braun, K. Wilkins, J. E. Balow, and H. A. Austin, "Membranous Nephropathy: Pilot Study of a Novel Regimen Combining Cyclosporine and Rituximab," Kidney International Reports, vol. 1, no. 2, pp. 73-84, 2016.

[53] L. F. Quintana, M. Blasco, M. Seras et al., "Antiphospholipase A2 receptor antibody levels predict the risk of posttransplantation recurrence of membranous nephropathy," Transplantation, vol. 99, no. 8, pp. 1709-1714, 2015.

[54] G. Gupta, H. Fattah, R. Ayalon et al., "Pre-transplant phospholipase $\mathrm{A} 2$ receptor autoantibody concentration is associated with clinically significant recurrence of membranous nephropathy post-kidney transplantation," Clinical Transplantation, vol. 30, no. 4, pp. 461-469, 2016.

[55] B. Seitz-Polski, C. Payré, D. Ambrosetti et al., "Prediction of membranous nephropathy recurrence after transplantation by monitoring of anti-PLA2R1 (M-type phospholipase A2 receptor) autoantibodies: A case series of 15 patients," Nephrology Dialysis Transplantation, vol. 29, no. 12, pp. 2334-2342, 2014.

[56] A. Kattah, R. Ayalon, L. H. Beck Jr. et al., "Anti-phospholipase $\mathrm{A}_{2}$ receptor antibodies in recurrent membranous nephropathy," American Journal of Transplantation, vol. 15, no. 5, pp. 13491359, 2015.

[57] A. Behnert, M. J. Fritzler, B. Teng et al., "An anti-phospholipase $\mathrm{A}_{2}$ receptor quantitative immunoassay and epitope analysis in membranous nephropathy reveals different antigenic domains of the receptor," PLoS ONE, vol. 8, no. 4, Article ID e61669, 2013.

[58] M. Fresquet, T. A. Jowitt, and J. Gummadova, "Identification of a major epitope recognized by PLA2R autoantibodies in primary membranous nephropathy," Journal of the American Society of Nephrology, vol. 26, no. 2, pp. 302-313, 2015.

[59] L. Kao, V. Lam, M. Waldman, R. J. Glassock, and Q. Zhu, "Identification of the immunodominant epitope region in phospholipase A2 receptor-mediating autoantibody binding in idiopathic membranous nephropathy," Journal of the American Society of Nephrology, vol. 26, no. 2, pp. 291-301, 2015.

[60] L. H. Beck, "The dominant humoral epitope in phospholipase a2 receptor-1: Presentation matters when serving up a slice of $\pi$," Journal of the American Society of Nephrology, vol. 26, no. 2, pp. 237-239, 2015.

[61] B. Seitz-Polski, G. Dolla, C. Payre et al., "Epitope Spreading of Autoantibody Response to PLA2R Associates with Poor Prognosis in Membranous Nephropathy," Journal of the American Society of Nephrology, vol. 27, no. 5, pp. 1517-1533, 2016.

[62] H. C. Stanescu, M. Arcos-Burgos, A. Medlar et al., "Risk HLA-DQA1 and $\mathrm{PLA}_{2} \mathrm{R} 1$ alleles in idiopathic membranous nephropathy," The New England Journal of Medicine, vol. 364, no. 7, pp. 616-626, 2011.

[63] G. Bullich, J. Ballarín, A. Oliver et al., "HLA-DQA1 and PLA2R1 polymorphisms and risk of idiopathic membranous nephropathy," Clinical Journal of the American Society of Nephrology, vol. 9, no. 2, pp. 335-343, 2014.

[64] P. Sekula, Y. Li, H. C. Stanescu et al., "Genetic risk variants for membranous nephropathy: extension of and association with other chronic kidney disease aetiologies," Nephrology Dialysis Transplantation, vol. 32, no. 2, pp. 325-332, 2017.

[65] M. Saeed, M. L. Beggs, P. D. Walker, and C. P. Larsen, "PLA2Rassociated membranous glomerulopathy is modulated by common variants in PLA2R1 and HLA-DQA1 genes," Genes and Immunity, vol. 15, no. 8, pp. 556-561, 2014.

[66] C. P. Larsen, M. L. Beggs, P. D. Walker, M. Saeed, J. M. Ambruzs, and N. C. Messias, "Histopathologic effect of apoll risk alleles in PLA2r-associated membranous glomerulopathy," American Journal of Kidney Diseases, vol. 64, no. 1, pp. 161-163, 2014.

[67] J. Lv, W. Hou, X. Zhou et al., "Interaction between PLA2R1 and HLA-DQA1 variants associates with anti-PLA2R antibodies and membranous nephropathy," Journal of the American Society of Nephrology, vol. 24, no. 8, pp. 1323-1329, 2013.

[68] W. Le, J. Shi, T. Zhang et al., "HLA-DRB1*15:01 and HLADRB3 $^{\star} 02: 02$ in PLA2R-Related Membranous Nephropathy," Journal of the American Society of Nephrology, vol. 28, no. 5, pp. 1642-1650, 2017.

[69] Z. Cui, L. Xie, F. Chen et al., "MHC Class II Risk Alleles and Amino Acid Residues in Idiopathic Membranous Nephropathy," Journal of the American Society of Nephrology, vol. 28, no. 5, pp. 1651-1664, 2017.

[70] M. Thiri, K. Honda, K. Kashiwase et al., "High-density Association Mapping and Interaction Analysis of PLA2R1 and HLA Regions with Idiopathic Membranous Nephropathy in Japanese," Scientific Reports, vol. 6, Article ID 38189, 2016.

[71] M. J. H. Coenen, J. M. Hofstra, H. Debiec et al., "Phospholipase A2 receptor (PLA2R1) sequence variants in idiopathic membranous nephropathy," Journal of the American Society of Nephrology, vol. 24, no. 4, pp. 677-683, 2013.

[72] C. D. Short, J. Feehally, R. Gokal, and N. P. Mallick, "Familial membranous nephropathy.," BMJ, vol. 289, no. 6457, pp. 15001500, 1984.

[73] L. Lin, W. M. Wang, X. X. Pan et al., "Biomarkers to detect membranous nephropathy in Chinese patients," Oncotarget, vol. 7, no. 42, pp. 67868-67879, 2016.

[74] C. P. Larsen, L. N. Cossey, and L. H. Beck, "THSD7A staining of membranous glomerulopathy in clinical practice reveals cases with dual autoantibody positivity," Modern Pathology, vol. 29, no. 4, pp. 421-426, 2016. 
[75] E. Hoxha, T. Wiech, P. R. Stahl et al., "A mechanism for cancerassociated membranous nephropathy," New England Journal of Medicine, vol. 374, no. 20, pp. 1995-1996, 2016.

[76] E. Hoxha, L. H. Beck, T. Wiech et al., "An Indirect Immunofluorescence Method Facilitates Detection of Thrombospondin Type 1 Domain-Containing 7A-Specific Antibodies in Membranous Nephropathy," Journal of the American Society of Nephrology, vol. 28, no. 2, pp. 520-531, 2017.

[77] N. M. Tomas, E. Hoxha, A. T. Reinicke et al., "Autoantibodies against thrombospondin type 1 domain-containing $7 \mathrm{~A}$ induce membranous nephropathy," Journal of Clinical Investigation, vol. 126, no. 7, pp. 2519-2532, 2016.

[78] R. Ayalon and L. H. Beck, "Membranous nephropathy: not just a disease for adults," Pediatric Nephrology, vol. 30, no. 1, pp. 3139, 2015.

[79] S. Menon and R. P. Valentini, "Membranous nephropathy in children: Clinical presentation and therapeutic approach," Pediatric Nephrology, vol. 25, no. 8, pp. 1419-1428, 2010.

[80] H. Debiec, V. Guigonis, B. Mougenot et al., "Brief report: Antenatal membranous glomerulonephritis due to anti-neutral endopeptidase antibodies," New England Journal of Medicine, vol. 346, no. 26, pp. 2053-2060, 2002.

[81] J. L. Nortier, H. Debiec, Y. Tournay et al., "Neonatal disease in neutral endopeptidase alloimmunization: Lessons for immunological monitoring," Pediatric Nephrology, vol. 21, no. 10, pp. 1399-1405, 2006.

[82] M. Vivarelli, F. Emma, T. Pellé et al., "Genetic homogeneity but IgG subclass-dependent clinical variability of alloimmune membranous nephropathy with anti-neutral endopeptidase antibodies," Kidney International, vol. 87, no. 3, pp. 602-609, 2015.

[83] D. H. Sandberg, C. W. Bernstein, R. M. McIntosh, R. Carr, and J. Strauss, "Severe steroid-responsive nephrosis associated with hypersensitivity," The Lancet, vol. 309, no. 8008, pp. 388-391, 1977.

[84] M. Abdel-Hafez, M. Shimada, P. Y. Lee, R. J. Johnson, and E. H. Garin, "Idiopathic Nephrotic Syndrome and Atopy: Is There a Common Link?" American Journal of Kidney Diseases, vol. 54, no. 5, pp. 945-953, 2009.

[85] H. Debiec, F. Lefeu, M. J. Kemper et al., "Early-childhood membranous nephropathy due to cationic bovine serum albumin," New England Journal of Medicine, vol. 364, no. 22, pp. 2101-2110, 2011.

[86] M. Prunotto, M. L. Carnevali, G. Candiano et al., "Autoimmunity in membranous nephropathy targets aldose reductase and SOD2," Journal of the American Society of Nephrology, vol. 21, no. 3, pp. 507-519, 2010.

[87] M. Bruschi, M. L. Carnevali, C. Murtas et al., "Direct characterization of target podocyte antigens and auto-antibodies in human membranous glomerulonephritis: Alfa-enolase and borderline antigens," Journal of Proteomics, vol. 74, no. 10, pp. 2008-2017, 2011.

[88] C. Murtas, M. Bruschi, G. Candiano et al., "Coexistence of different circulating anti-podocyte antibodies in membranous nephropathy," Clinical Journal of the American Society of Nephrology, vol. 7, no. 9, pp. 1394-1400, 2012.

[89] Y. Kimura, N. Miura, H. Debiec et al., "Circulating antibodies to $\alpha$-enolase and phospholipase A2 receptor and composition of glomerular deposits in Japanese patients with primary or secondary membranous nephropathy," Clinical and Experimental Nephrology, vol. 21, no. 1, pp. 117-126, 2017. 


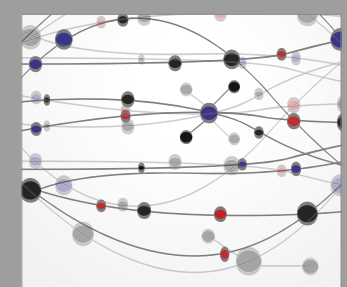

The Scientific World Journal
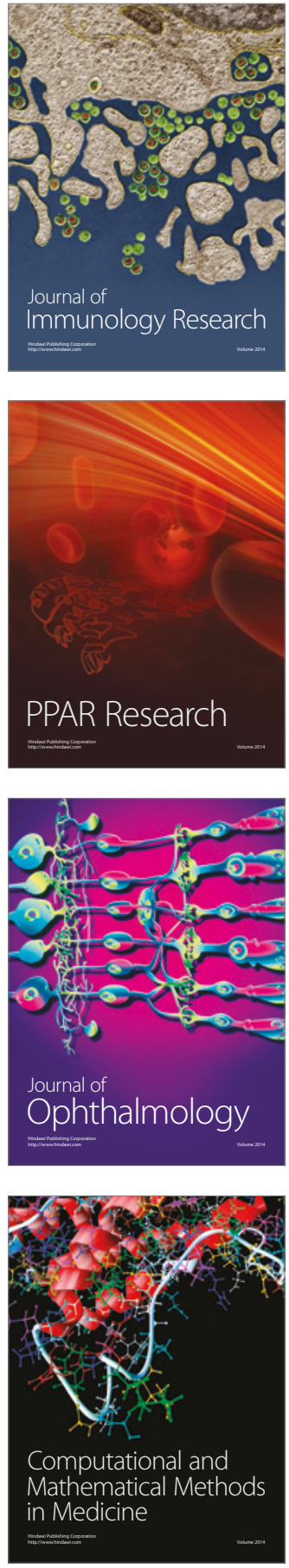

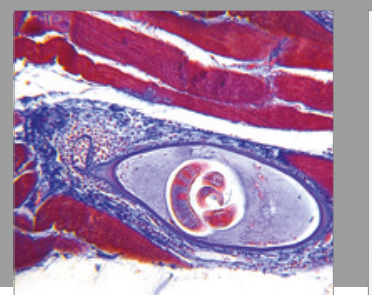

Gastroenterology Research and Practice
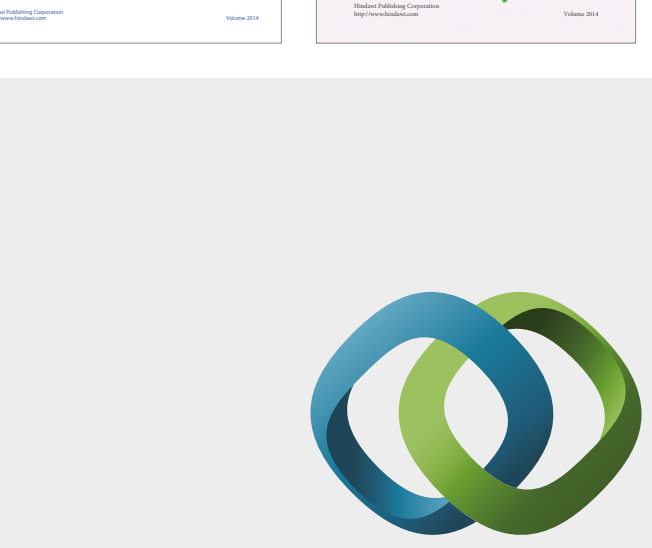

\section{Hindawi}

Submit your manuscripts at

https://www.hindawi.com
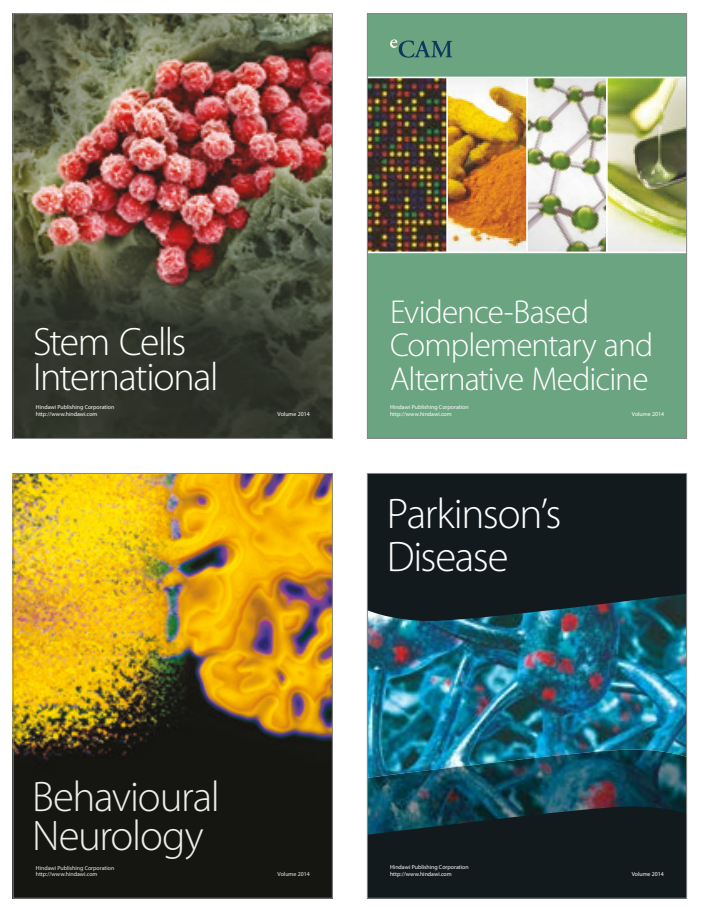
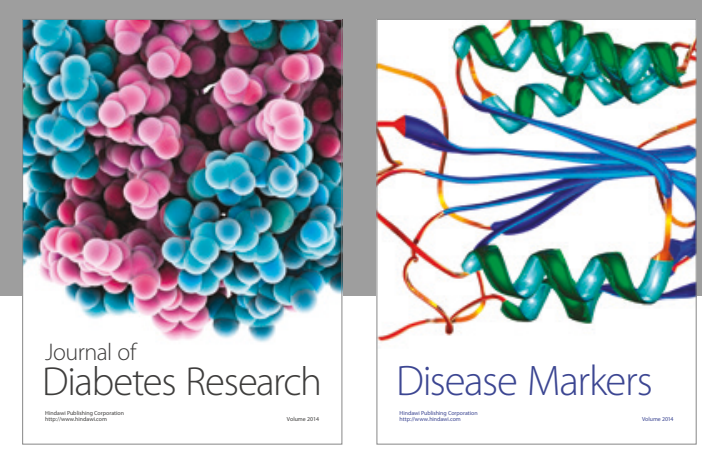

Disease Markers
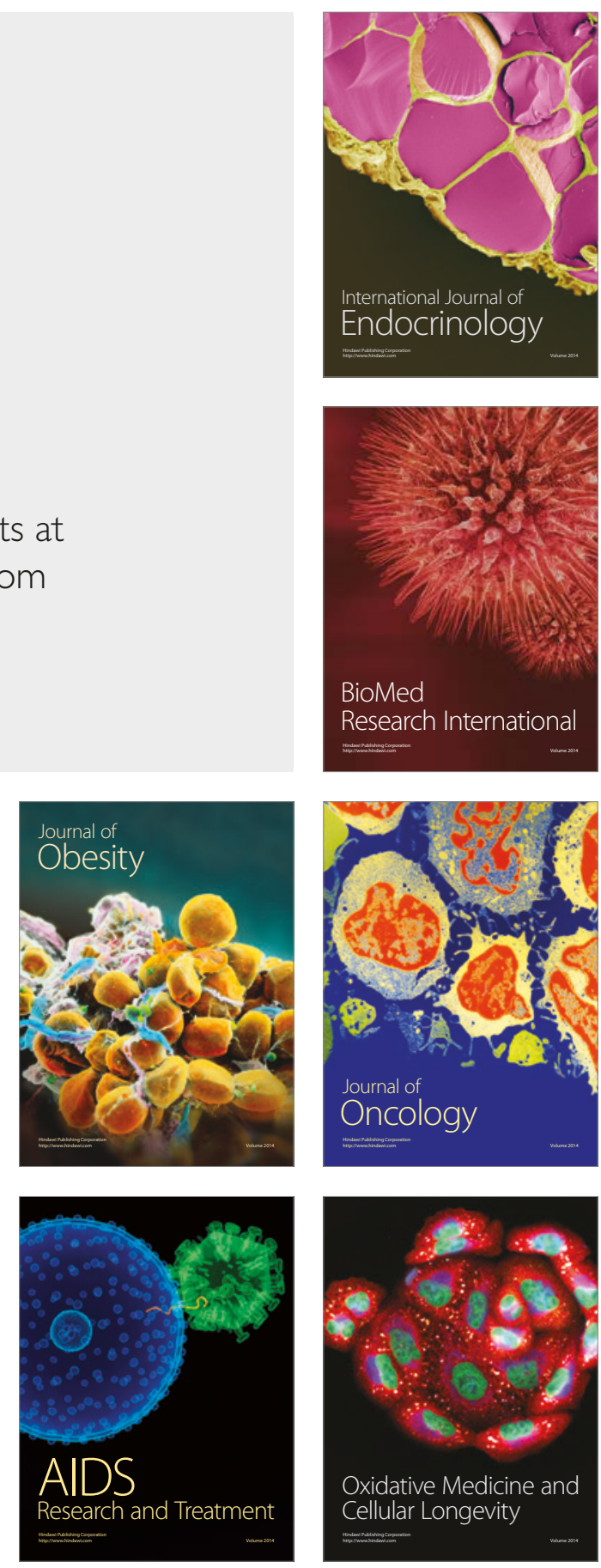